\title{
The fish that beat physics
}

\author{
Silvery fish have evolved an elegant optical scheme for overcoming the Brewster effect, creating \\ broadband, polarization-neutral reflections for any angle of incidence. Nicholas Roberts explained to \\ Nature Photonics how and why they do it.
}

\begin{abstract}
What exactly have you discovered regarding reflections from silvery fish? Dielectric multilayer mirrors are common in nature and are found in various animals, for example iridescent beetles, other insects, crustaceans and fish. In fish, multilayer reflectors consist of alternating layers of high-refractive-index guanine crystals and low-refractive-index cytoplasm. Up to this point, it was believed that such multilayer reflectors should exhibit normal Fresnel-type behaviour, and fully polarize on reflection at Brewster's angle. Associated with this polarization response is a drop in mean reflectivity - the 'Brewster window' effect. We have discovered a generic and novel optical mechanism in silvery fish like herring, sardine and sprat that seemingly breaks this basic law of reflection, enabling nonpolarizing reflections to occur.
\end{abstract}

Why is polarization-neutral, broadband reflectivity important for such fish? The simple answer is camouflage. Silvery fish generally live in the mid-water pelagic zone where the radiance distribution of light is symmetric about the vertical axis. Optimal concealment from predators requires broadband, high-percentage reflectivity over all angles of incidence. The problem with normal multilayer reflectors is that as you approach Brewster's angle, the reflectivity drops and you lose the camouflage. However, polarization neutrality is a way of avoiding the reflectivity drop across the Brewster window. What we have discovered in these species of fish is that their mechanism of camouflage exhibits polarization neutrality, thus maximizing their reflectivity over all angles. This would help the fish best match the open-water background light field and aid their ability to camouflage themselves against predators.

Please describe the fish structure and the optical mechanisms involved.

Fish skin is made up of several layers. Underneath the scales is a layer of tissue called the stratum argenteum. This layer is made up of the multiple layers of highindex guanine and low-index cytoplasm. Guanine itself has an exceptionally large birefringence (one of the highest of any

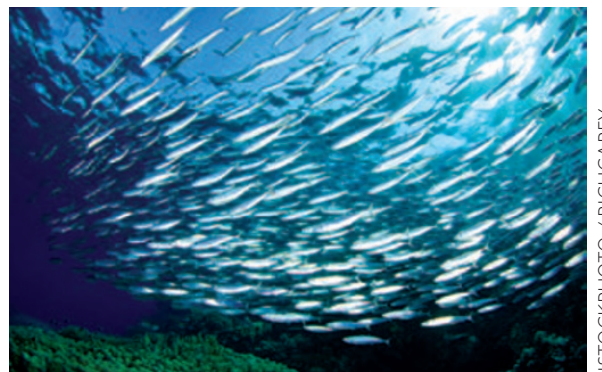

for example in optical fibres, LED back reflectors and dielectric waveguides. As the refractive index and birefringence of the guanine crystals are similar to those of some highly birefringent polymers, we anticipate that a synthetic biomimetic device could be developed for applications where multilayer mirrors are made from polymers. In addition, modern deposition techniques can control the birefringence of layers so I am sure that building this type of

Silvery fish like sardines, sprat and mackerel use birefringence to help them camouflage themselves in oceanic water.

natural material), so as light travels through it different polarizations experience different refractive indices. These are 1.83 for two polarization axes and 1.46 for the other. What we have discovered is that there is not just one type of guanine crystal but actually two, the difference between them being a rotational transformation of the refractive indices by $90^{\circ}$. In some of the crystals the axis with index 1.46 points out of the plane of the layers and in others it lies within the plane. The result is different Brewster's angles for the two types of crystal, and by mixing these two types the multilayer structure has a range of Brewster's angles (ranging from approximately $33^{\circ}$ to $67^{\circ}$ ). Overall, it does not matter what angle light is incident at; some of the polarizations are always reflected from some of the layers. Our work suggests that by having a particular mixing ratio of these two types of guanine crystal, these species of fish have evolved a structure that enables near-constant reflectivity over all angles of incidence. This creates an optimal solution for camouflage purposes.

How could such a capability be put to good use in the world of optical engineering? In principle, the mechanism could find applications wherever high-percentage, polarization-independent reflectivity is required over all angles of incidence. Multilayer reflectors are one of the most commonly used optical devices in industry. There are many examples where this property is of fundamental importance, structure is possible. Alternatively, you could create a holey structure that you fill with liquid crystal, which has an electronically controllable birefringence.

What are the advantages of nature's approach compared with man-made designs?

Common to all previously described nonpolarizing mirror designs is the exploitation of a difference in refractive index between the low-index layers in the reflector and the external environment. This enables the reflector to be screened from angles of incidence where polarization occurs. Unlike existing non-polarizing reflectors, a biomimetic design based on the optical mechanism in these fish would permit the environmental medium and the low-index layers to be made from the same material, and this would provide an advantage in applications where the thermal and mechanical properties of the reflector benefit from being constructed from the same material as the external surroundings.

What are your future plans for research in this area?

Many aquatic animals, such as squid, cuttlefish and mantis shrimp, are sensitive to the polarization of light and have welldeveloped polarization vision. We are very interested in the polarization properties of reflectors that they use and any novel optics that have evolved as a result of evolutionary adaptations.

INTERVIEW BY OLIVER GRAYDON Nicholas Roberts and co-workers have a Letter on the reflectivity properties and mechanisms of silvery fish at http://dx.doi. org/10.1038/nphoton.2012.260. 